\title{
Anhaltende Produktionslücken durch Vorleistungsengpässe
}

\author{
Die anhaltenden Probleme bei der Beschaffung von Vorleistungen belasten die deutsche \\ Wirtschaft. Drei Viertel der Unternehmen berichten derzeit von Produktionsausfällen von \\ im Durchschnitt $7 \%$. Erst ab dem zweiten Halbjahr 2022 wird sich die Situation deutlich \\ verbessern. Aber auch im Jahr 2023 rechnet noch knapp die Hälfte der privatwirtschaftlichen \\ Unternehmen mit Ausfällen. Neben den fehlenden Vorleistungen ist vor allem der \\ Fachkräftemangel eine permanente Produktionsbremse.
}

Der Mangel an Vorleistungen hat sich nach dem wirtschaftlichen Tiefpunkt der Coronakrise im Frühjahr 2020 zu einer wesentlichen Bremse für den Aufschwung entwickelt (Bardt et al., 2021a). Elektronische Bauteile, Baumaterialien, Metalle, Chemikalien und Papier - die Liste der Vorprodukte mit Lieferschwierigkeiten ist lang. Teilweise können die notwendigen Teile zu höheren Preisen beschafft werden, damit die eigene Produktion am Laufen gehalten wird. Vor allem der Automobilindustrie macht der Mangel an Halbleitern und elektronischen Bauteilen schwer zu schaffen. Die Produktion lag dort zuletzt auf Basis des Produktionsindexes um rund $50 \%$ unter dem Niveau des Jahres 2018. Dies wirkt über die vielfältigen Verflechtungen in andere Wirtschaftsbereiche hinein, die ihrerseits unter Transportproblemen und Produktionsbehinderungen leiden. Nachdem die durch die Pandemie bedingte Produktionslücke (gegenüber dem Jahresdurchschnitt 2019) der gesamten deutschen Industrie in Höhe von gut $30 \%$ im Frühjahr 2020 bis zum Jahresende 2020 auf knapp $4 \%$ verringert werden konnte, ist sie im Jahresverlauf 2021 wieder deutlich auf zuletzt rund $10 \%$ angewachsen. Diese Produktionslücke steht einem weiterhin wachsenden Auftragsbestand gegenüber.

Die Ursachen für diese Störungen in den internationalen Vorleistungsnetzwerken sind vielfältig:

- Die überraschend schnelle Erholung der Weltwirtschaft nach dem Tiefpunkt im Frühjahr 2020 hat zu einem Rückstau geführt. Gleichzeitig müssen sich Zulieferund Wertschöpfungsstrukturen nach dem plötzlichen Stillstand von 2020 und dem Stop-and-Go im Gefolge der weiteren Infektionswellen neu organisieren.

(c) Der/die Autor:in 2022. Open Access: Dieser Artikel wird unter der Creative Commons Namensnennung 4.0 International Lizenz veröffentlicht (creativecommons.org/licenses/by/4.0/deed.de).

Open Access wird durch die ZBW - Leibniz-Informationszentrum Wirtschaft gefördert.
- Das weltweit anhaltende Pandemie-Geschehen sorgt - etwa über die Schließung von Häfen oder fehlende Schiffsbesatzungen - nach wie vor für Störungen im internationalen Warenhandel. Einzelereignisse in der Logistik wie die Sperrung des Suezkanals verschärften zwischenzeitlich den Rückstau. Direkte Produktionsausfälle durch die Pandemie kommen hinzu.

- Ein deutlicher Nachfrageanstieg auf dem Markt für Halbleiter - durch eine coronabedingte Mehrnachfrage etwa für Laptops und durch technologische Mehrbedarfe für Elektroautos - trifft auf hoch ausgelastete und erst mittelfristig erweiterbare Angebotskapazitäten.

- Temporäre Angebotsausfälle, beispielsweise durch den Brand von Halbleiterfabriken in Asien oder auf dem Holzmarkt nach Waldbränden in den USA, haben die Engpässe zusätzlich verschärft.

Diese Produktionsprobleme bei den Unternehmen führen zu Versorgungsengpässen auf bestimmten Märkten, zu höheren Produktionskosten und teilweise zu höheren Verkaufspreisen. Im Sommer 2021 sah rund die Hälfte

Prof. Dr. Hubertus Bardt leitet den Bereich

Wissenschaft am Institut der deutschen Wirtschaft (IW) in Köln und ist Honorarprofessor an der Heinrich-HeineUniversität Düsseldorf.

Prof. Dr. Michael Grömling leitet die Forschungsgruppe Konjunktur am Institut der deutschen Wirtschaft (IW) in Köln und ist Professor für Volkswirtschaftslehre an der International University (IU), Campus Köln. 
der Unternehmen hohe oder mittlere Überwälzungsmöglichkeiten dieser höheren Produktionskosten auf ihre Verkaufspreise (Bardt et al., 2021b). Dies hat aber auch zur Folge, dass sich zum einen die Nachfrage in diesen Märkten nach den Einschränkungen infolge des Einbruchs 2020 nicht vollständig erholen kann. Zum anderen gehen die nicht überwälzbaren Produktionskostenanstiege zulasten der Rentabilität und dies dämpft die Erholung der Investitionen deutlich ab. Seit dem dritten Quartal 2020 war mit Blick auf die Ausrüstungsinvestitionen in Deutschland kein Fortschritt mehr zu beobachten (Bardt et al., 2021a).

\section{Differenzierte Erholungszeiten}

Die aufgezeigten Ursachen der gegenwärtigen Engpässe bei den Vorleistungen deuten auf unterschiedliche Zeiträume hin, in denen eine Normalisierung der Produktionsprozesse stattfinden kann. Kurzfristige Vorleistungs- und Angebotsausfälle können zumeist zeitnah ausgeglichen werden. Das gilt auch für Einzelereignisse in der Logistik wie Bahnstreiks oder die Suez-Blockade, die gleichwohl in der gegenwärtig angespannten Situation stärker wirken als im Normalfall. Die Nachwirkungen der Coronakrise 2020 in Logistik und Produktion sollten ebenfalls schrittweise zurückgehen. Mit kurz- und mittelfristigen Ausfällen aufgrund von erneuten und unerwartet starken CoronaAusbrüchen muss gleichwohl weiterhin gerechnet werden.

Bei den Halbleitern ist eine kurzfristige Verbesserung dagegen nicht zu erwarten (SIA, 2021). Der zusätzliche Nachfrageanstieg konnte nicht durch freie Kapazitäten aufgefangen werden. Auch eine kurzfristige Kapazitätsausweitung ist aufgrund der hohen Kosten und der notwendigen Planungsvorläufe nicht möglich. Eine Entspannung könnte allenfalls über die Nachfrageseite erfolgen: Mehranschaffungen als direkte Folge der Coronakrise werden nicht erneut vorgenommen. Zudem dämpfen höhere Preise die Nachfrage. Perspektivisch dürften die verfügbaren Chips in wertschöpfungsintensiven Gütern wie Kraftfahrzeugen eingesetzt werden. Hier liegt allerdings mit Blick auf Deutschland und die hohe Bedeutung der Automobilwirtschaft im gesamtwirtschaftlichen Branchengefüge ein großes engpassbedingtes Konjunkturrisiko (Bardt et al., 2021a).

\section{Betroffenheit der Unternehmen}

Um die bestehenden und die in naher Zukunft erwarteten Produktionsausfälle in der deutschen Wirtschaft zu vermessen, wurden im Rahmen der IW-Konjunkturumfrage (Grömling, 2018) im November 2021 über 2.800 Unternehmen nach dem Ausmaß und den Gründen ihrer Produktionsbeeinträchtigungen befragt. Zunächst ist festzuhalten, dass für 2022 eine hohe konjunkturelle Zuversicht dominiert (Grömling, 2021). Fast die Hälfte der Unterneh-

\section{Abbildung 1}

Ausmaß der Produktionsbeeinträchtigungen wegen Vorleistungsproblemen und Produktionslücke

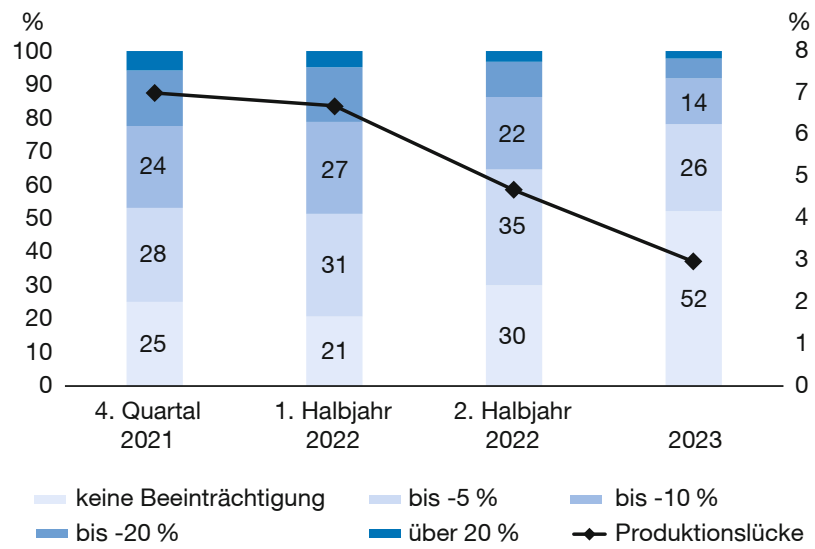

Anmerkungen: Anteil auf Basis ungewichteter Ergebnisse der IW-Konjunkturbefragung unter 2.842 Unternehmen in Deutschland im November 2021. Produktionslücke (Produktionsausfall im Vergleich zur Normalproduktion vor der Coronapandemie) auf Basis gewichteter Ergebnisse (nach Betrieben, Beschäftigten und Regionen).

Quelle: Institut der deutschen Wirtschaft.

men erwartet eine höhere Produktion oder Geschäftstätigkeit als im Vorjahr, nur $15 \%$ aller Firmen erwarten einen Rückgang gegenüber 2021. Gleichwohl konstatieren die vom IW befragten Unternehmen anhaltende Produktionsausfälle - in zeitlich unterschiedlicher Intensität:

Bezogen auf das vierte Quartal 2021 stellen drei Viertel der befragten Unternehmen (auf Basis von ungewichteten Ergebnissen) Produktionsausfälle infolge fehlender Vorleistungen fest (vgl. Abbildung 1). Davon gehen 28 Prozentpunkte in einer Größenordnung von bis zu $5 \%$ an Produktionseinschränkung aus, weitere 24 Prozentpunkte von Einbußen bis zu $10 \%$. Knapp ein Viertel der Betriebe hatte im vierten Quartal 2021 mit großen Problemen zu kämpfen - dabei schätzen $17 \%$ aller befragten Firmen inre Produktionsausfälle auf bis zu $20 \%$ und $6 \%$ auf über $20 \%$. Gut $1 \%$ der Betriebe produzierte weniger als die Hälfte seines Normaloutputs. Die stärksten Produktionseinschränkungen erfahren derzeit die Industrieunternehmen - darunter vor allem die Hersteller von Investitionsgütern. Nur ein Zehntel der Investitionsgüterfirmen konnte im vierten Quartal 2021 reibungslos produzieren - im Dienstleistungssektor waren es dagegen fast $44 \%$.

Die IW-Befragung zeigt, dass gestörte Produktionsprozesse die Unternehmen auch 2022 die Konjunktur hierzulande belasten werden. Während im Vergleich zum vierten Quartal 2021 das Belastungsniveau im ersten Halbjahr 2022 sogar leicht zunehmen dürfte, ist in der zweiten Jahreshälfte 2022 eine spürbare Entlastung zu erwarten: Der Anteil der Betriebe ohne Beeinträchtigung steigt zunächst auf knapp $80 \%$ 
und geht dann auf $70 \%$ zurück. Rund ein Drittel der Betriebe rechnet aber auch in der zweiten Jahreshälfte 2022 mit Produktionsausfällen in einer Größenordnung von mehr als $5 \%$ davon rund 3 Prozentpunkte mit Einbußen jenseits von $20 \%$.

Eine weitgehende mittelfristige Entspannung der Zulieferund Produktionsprobleme und der ihr zugrunde liegenden Ursachen erwartet vor dem Hintergrund der aktuellen Informationslage und der damit einhergehenden Verunsicherungen offensichtlich nur gut die Hälfte der befragten Firmen. Gut ein Viertel geht auch für 2023 davon aus, dass Produktionsausfälle von bis zu $5 \%$ des Normaloutputs eintreten. Gut $2 \%$ aller Betriebe erwarten sogar einen Ausfall von über $20 \%$. Diese relative Entspannung gilt sowohl für die Dienstleister (mit ihrem aktuell deutlich geringeren Belastungsniveau) als auch für die Industrie.

\section{Ausmaß der Produktionslücken}

In Abbildung 1 sind die Anteile von Unternehmen dargestellt, die am aktuellen Rand und für die nähere Zukunft ein bestimmtes Ausmaß an Produktionseinbußen infolge von gestörten Zulieferketten erwartet. Dabei werden die Unternehmen unabhängig von ihrer Betriebsgröße berücksichtigt. Um eine Größenordnung für die damit einhergehenden gesamtwirtschaftlichen Produktionslücken zu erhalten, wird auf gewichtete Ergebnisse zurückgegriffen. Da in der Befragung kleine Unternehmen (weniger als zehn Beschäftigte) nicht berücksichtigt werden, halten sich die Unterschiede zwischen gewichteten und ungewichteten Ergebnissen in engen Grenzen. Zudem muss bei der Schätzung einer gesamtwirtschaftlichen Produktionslücke das Intervall hinsichtlich der Betroffenheit normiert werden. Dazu wurde die Mitte des jeweiligen Intervalls gewählt.

Auf Basis dieser Schätzungen und der vom IW befragten Wirtschaftsbereiche ergibt sich für das vierte Quartal 2021 eine Produktionslücke in den der Befragung zugrunde liegenden Wirtschaftsbereichen - Industrie, Bauwirtschaft und Teile der privatwirtschaftlichen Dienstleister - in Höhe von $7 \%$. Gemäß dieser Schätzung gehen die gesamtwirtschaftlichen Produktionsausfälle im ersten Halbjahr 2022 auf nur knapp $7 \%$ zurück und im zweiten Halbjahr 2022 dürften sie sich auf knapp $5 \%$ belaufen.

Abbildung 1 zeigt, dass auch im Jahr 2023 die angebotsseitigen Störungen im gegenwärtigen Urteil der Unternehmen nicht behoben sein werden (Leiss und Wohlrabe, 2021). Die damit einhergehende Produktionslücke dürfte dann schätzungsweise bei $3 \%$ im Vergleich mit einer $\mathrm{Ge}$ schäftslage ohne Vorleistungs- und Produktionsstörungen liegen. Dabei ist zu bedenken, dass in der IW-Konjunkturumfrage der gesamte öffentliche Sektor und Teile der privatwirtschaftlichen Dienstleister (wie das Finanzwesen)

\section{Abbildung 2}

Gründe von Produktionsproblemen

Anteil von Unternehmen, die starke oder mittlere Produktionsbeeinträchtigungen für 2022 erwarten, in \% aller Unternehmen

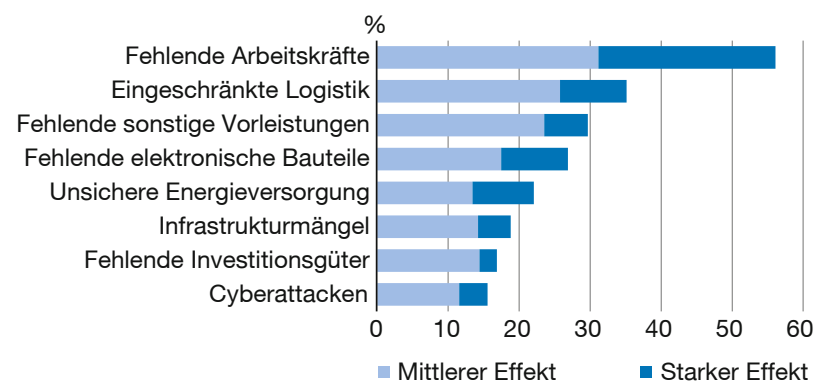

Anmerkungen: Anteil auf Basis ungewichteter Ergebnisse der IW-Konjunkturbefragung unter 2.842 Unternehmen in Deutschland im November 2021.

Quelle: Institut der deutschen Wirtschaft.

nicht berücksichtigt werden. Vielmehr dominieren unternehmensnahe Dienstleister sowie Industrie- und Bauunternehmen, wo die Produktionsbeeinträchtigungen aufgrund ihrer arbeitsteiligen Produktionsprozesse stärker auftreten.

\section{Weitere Einschränkungen}

Fehlende Vorleistungen sind nur ein Grund für Produktionsausfälle in Unternehmen in Deutschland (vgl. Abbildung 2). Gemäß der IW-Umfrage erwarten $27 \%$ der Unternehmen starke oder mittlere Beeinträchtigungen ihrer Produktion im Jahr 2022 durch fehlende elektronische Bauteile wie Halbleiter. Vor allem bei der Produktion von Investitionsgütern sind hier große Störungen angezeigt: Über $47 \%$ dieser Betriebe sieht auch 2022 starke oder mittlere Beeinträchtigungen durch eine nicht ausreichende Belieferung mit elektronischen Bauteilen auf sich zukommen. $30 \%$ aller befragten Firmen sind durch einen Mangel an sonstigen Vorleistungen beeinträchtigt. Das gilt für die Industrie und die Bauwirtschaft gleichermaßen.

Die aktuellen Produktionsstörungen infolge fehlender Zulieferungen resultieren auch aus Logistikproblemen. Dies trifft die Unternehmen sowohl bei der Zulieferung von Vorleistungen als auch bei der eigenen Belieferung von Kunden. Mit Blick auf 2022 erwarten 35\% der Befragten mittlere oder starke Probleme in der Produktion durch Logistikengpässe - darunter 10 Prozentpunkte in einem starken Ausmaß. Auch hier sind die größten Einschränkungen im Bereich der Industrie zu verorten. Während nur ein Fünftel der Industriefirmen ohne Logistikprobleme agieren kann, sind es in der Bauwirtschaft gut 30\% und im Dienstleistungsbereich die Hälfte der vom IW befragten Unternehmen. In diesem Kontext können auch Infrastrukturprobleme in Deutschland aufgeführt werden. Knapp ein Fünftel aller Unternehmen führt Produktionsbeeinträchtigungen 2022 auf Infrastruk- 
turmängel zurück. Dabei tragen zum einen die Verkehrsbeschränkungen bei - anekdotisch erkennbar an mehreren Brückensperrungen in Deutschland. Zum anderen können hier auch die allgemeinen Schwierigkeiten mit der digitalen Infrastruktur genannt werden. Eine unsichere Energieversorgung stellt für die Geschäftsabläufe in den Unternehmen ein unterschiedlich hohes Produktionsrisiko dar. Insbesondere die energieintensiven Produktionsprozesse in der Industrie dürften durch eine stockende Energieversorgung stark belastet werden. Jedenfalls sehen $23 \%$ der Industriebetriebe mögliche starke oder mittlere Beeinträchtigungen - im Dienstleistungssektor liegt der entsprechende Anteil aber auch bei $19 \%$. Die Informations- und Kommunikationstechnologien, die hier breitflächig zur Anwendung kommen, erfordern ebenfalls eine hohe Versorgungssicherheit. Immerhin $16 \%$ aller Unternehmen messen auch den Produktionsausfällen infolge von Cyberattacken eine starke oder mittlere Bedeutung zu. Hier sind es insbesondere die Dienstleister, die mit Blick auf das Jahr 2022 von einer erhöhten Vorsicht geprägt sind.

Die gegenwärtig schwache Investitionstätigkeit in Deutschland ist zum einen von der bestehenden Verunsicherung durch die Coronapandemie geprägt. Die Erholung und die Bedienung des mittlerweile aufgestauten Investitionsbedarfs in Deutschland wird allerdings auch durch eine eingeschränkte Verfügbarkeit von Investitionsgütern zurückgehalten (Bardt et al., 2021a). Gemäß der IW-Konjunkturumfrage vom November 2021 sprechen $17 \%$ der Unternehmen von Produktionsproblemen im Jahr 2022, weil sie nicht in ausreichendem Niveau an Investitionsgüter kommen. Dies gilt in besonderem Ausmaß für die Bauwirtschaft und im Dienstleistungssektor für den Bereich Transport und Logistik. Hier dürften sich insbesondere die Produktionsprobleme in der Automobilwirtschaft zu einem Investitionshindernis entwickelt haben.

Mit Blick auf das Produktionspotenzial und die möglichen Auswirkungen der Pandemie kommt neben dem Investitionsgeschehen auch dem Faktor Arbeit eine entscheidende Bedeutung zu. $56 \%$ der befragten Unternehmen sehen für 2022 Produktionshemmnisse, weil Fachkräfte fehlen (DIHK, 2021). Davon sprechen sogar 25 Prozentpunkte von starken Produktionsstörungen. Diese Situation ist mittlerweile ein langfristiges Problem in Deutschland - das zudem auch die Investitionstätigkeit der Unternehmen zurückhält (Grömling, 2017).
Abbildung 1 hat gezeigt, dass sich die Produktionsstörungen infolge von fehlenden Vorleistungen 2022 nicht vollständig zurückbilden. Vielmehr dürften sie sogar über das Jahr hinaus anhalten, allerding in deutlich abgeschwächter Weise. Eine Abschwächung wird auch für die in Abbildung 2 dargestellten konkreten Produktionsstörungen von den Unternehmen erwartet. Besonders deutlich ist der Rückgang bei den Behinderungen infolge fehlender sonstiger Vorleistungen. Der Anteil der in starkem oder mittlerem Ausmaß beeinträchtigten Unternehmen ist mit Blick auf das Gesamtjahr 2022 um 8 Prozentpunkte niedriger als zum Befragungszeitpunkt im November 2021. Hier erwarten die Unternehmen eine schrittweise Auflösung der Engpässe. Gleiches gilt für die Logistikprobleme mit einem Minus von 4 Prozentpunkten. Bei Elektronikbauteilen wird dagegen mit kaum einer Verbesserung gerechnet. Dabei geht allerdings der Anteil der stark betroffenen Unternehmen deutlich von $15 \%$ auf $9 \%$ zurück und der Anteil der im mittleren Ausmaß beeinträchtigten Betriebe steigt entsprechend an. Das Halbleiterproblem dürfte nach Ansicht der Unternehmen also auch 2022 prägen. Zunehmende Sorgen gibt es hingegen bezüglich der Cyberattacken (+3 Prozentpunkte), hinsichtlich der Energieversorgung und durch fehlende Fachkräfte (jeweils 4 Prozentpunkte). Während die kurzfristigen Produktionshemmnisse abnehmen, ist bei den längerfristigen Unternehmensrisiken eine zunehmende Besorgnis zu beobachten.

\section{Literatur}

Bardt, H., M. Demary, M. Grömling, T. Hentze, M. Hüther, T. Obst, J. Pimpertz, T. Schaefer und H. Schäfer (2021a), Produktionsstörungen, Preiseffekte und Pandemie-Politik - IW-Konjunkturprognose Winter 2021, IW-Trends, 48(4), Online-Sonderausgabe, 2, 3-40.

Bardt, H., M. Diermeier, M. Grömling, M. Hüther und T. Obst (2021b), Lieferengpässe und Preisentwicklungen bei Rohstoffen und Vorleistungen. Corona Echo Effekte oder ,here to stay'?, IW-Report, 27.

DIHK (2021), Aufschwung droht ausgebremst zu werden - Standort Deutschland attraktiver für Unternehmen gestalten, DIHK-Konjunkturumfrage Herbst 2021.

Grömling, M. (2017), Was blockiert private Investitionen in Deutschland?, Wirtschaftspolitische Blätter, 64(3), 311-323.

Grömling, M. (2018), Methods and Applications of the IW business survey, IW-Report, 5.

Grömling, M. (2021), Zuversicht dominiert für 2022, IW-Konjunkturumfrage Spätherbst 2021, IW-Report, 45.

Leiss, F. und K. Wohlrabe (2021), Aktuelle Entwicklungen bei Materialengpässen und Lieferproblemen in der deutschen Wirtschaft, ifo Schnelldienst digital, 19.

SIA - Semiconductor Industry Association (2021), Comments on the Department of Commerce „Notice of Request for Public Comments on Risks in the Semiconductor Supply Chain“, https://www.semiconductors.org/wp-content/uploads/2021/11/SIA-Response-to-CommerceRFI-on-Semiconductor-Supply-Chain-Risks.pdf (11. Januar 2022).

Title: Persistent Production Gaps Due to Input Bottlenecks

Abstract: Ongoing problems with the procurement of intermediates are weighing on the German economy. Three-quarters of companies are currently reporting production losses averaging 7\%. The situation will not improve significantly until the second half of 2022. But even in 2023, just under half of private-sector companies still expect shortfalls. In addition to the lack of intermediates, the shortage of skilled workers is a permanent brake on production. 